\section{Fruit Texture Phenotypes of the RosBREED U.S. Apple Reference Germplasm Set}

\author{
Cari A. Schmitz, Matthew D. Clark, and James J. Luby ${ }^{1}$ \\ Department of Horticultural Science, University of Minnesota, 305 Alderman \\ Hall, 1970 Folwell Avenue, St. Paul, MN 55108
}

James M. Bradeen

Department of Plant Pathology, University of Minnesota, 495 Borlaug Hall, 1991 Upper Buford Circle, St. Paul, MN 55108

Yingzhu Guan and Katherine Evans

Washington State University Tree Fruit Research and Extension Center, 1100 N. Western Avenue, Wenatchee, WA 98801

\section{Benjamin Orcheski and Susan Brown}

Department of Horticulture, Cornell University, $630 \mathrm{~W}$. North Street, Geneva, NY 14456

\section{Sujeet Verma and Cameron Peace \\ Department of Horticulture, Washington State University, Pullman, WA 99164}

Additional index words. Malus $\times$ domestica, fruit quality, firmness, crispness, juiciness, penetrometer, sensory panel, marker-assisted breeding

\begin{abstract}
Establishing marker-locus-trait associations to enable marker-assisted breeding depends on having an extensive, reliable database for phenotypic traits of interest in relevant germplasm. A reference germplasm set of 467 apple (Malus $\times$ domestica Borkh.) cultivars, selections, and seedlings (referred to as individuals) was identified as part of the USDA-Specialty Crop Research Initiative (SCRI) project, RosBREED. The germplasm set provides efficient allelic representation of current parents in RosBREED demonstration apple breeding programs at Cornell University, Washington State University, and the University of Minnesota. Phenotyping at the three locations was conducted according to standardized protocols, focusing on fruit traits evaluated at harvest and after 10 and 20 weeks of refrigerated storage. Phenotypic data were collected for the sensory texture traits of firmness, crispness, and juiciness as well as for instrumental texture measures. In 2010 and 2011, fruit from 216 and 330 individuals, respectively, were harvested and a total of 369 individuals were evaluated over the two years. Correlations between sensory and instrumental texture measures were high in some instances. Moderate year-to-year repeatability of trait values was observed. Because each location had a largely unique set of individuals, as well as differing environmental conditions, means, ranges, and phenotypic variances differed greatly among locations for some traits. Loss of firmness and crispness during storage was more readily detected instrumentally than by the sensory evaluation.
\end{abstract}

Fruit texture traits, significant to apple breeder decision-making yet unobservable until tree maturity, are ideal candidates for marker-assisted breeding (MAB) and markerassisted selection (MAS). Marker-locus-trait associations, validated in germplasm relevant to a particular breeding program, facilitate MAB (Bliss, 2010). MAB is used to select parents with favorable alleles and MAS is

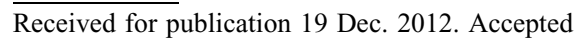
for publication 22 Jan. 2013.

Funded by the Specialty Crop Research Initiative Competitive Grant 2009-51181-05808 of the USDA's National Institute of Food and Agriculture.

${ }^{1}$ To whom reprint requests should be addressed; e-mail lubyx001@umn.edu.
Harker et al. (2003) predicted that fruit quality standards will evolve as consumers' expectations change. Speeding the breeding process through the use of molecular markers will aid apple breeders in developing higher quality fruit. A study using 'Red Delicious', 'Gala', and 'Braeburn' showed that in certain cultivars, firmness is of high importance to consumers, especially in combination with other fruit quality factors: firm apples, above a 53Newton threshold, can be improved on by changes in titratable acidity (TA) and soluble solids content (SSC), but soft apple acceptance cannot be improved on with changes in TA or SSC (Harker et al., 2008). These findings highlighted the use of genetic markers to select for fruit texture traits.

Studies of apple texture have used both sensory panels and instrumental measures (e.g., Evans et al., 2010; Ioannides et al., 2007; McKay et al., 2011; Zdunek et al., 2011). Differences in terms used to describe texture as well as their definitions make comparing sensory panel results difficult. For instance, the meaning of the term "crispness" differs across studies. Fillion and Kilcast (2002), using a trained sensory panel and a consumer panel, defined the term "crunchy" as describing lower-pitched sounds that continue throughout chewing, whereas "crisp" described a higher-pitched sound resulting from the clean split of the first bite. Both crisp and crunchy designations, when applied to food, express that the material breaks in the mouth rather than buckling or deforming. By studying sounds during biting dry and wet crisp foods, Vickers and Bourne (1976) defined the crispness sensation as a characteristic sound of a range of frequencies emitted during biting. For a thorough discussion of the crispness sensation, refer to Roudaut et al. (2002). In our study, described by Evans et al. (2012), "crispness" refers to the intensity of the cracking noise of the first bite. "Firmness" is equivalent to "hardness" and determined while chewing. "Juiciness" is expressed juice on chewing. A trained sensory panel, as small as three experienced individuals, has been shown to be reliable in a postharvest study of fruit texture (Brookfield et al., 2011). That panel was able to discern greater separation among cultivars than was achieved with instrumental measures. Although sensory panels more closely mimic consumer perception of fruit texture, they can be time-consuming and difficult to standardize.

Puncture tests, performed with various mechanized penetrometers, are typically used to determine firmness and juiciness (e.g., Harker et al., 2006). Harker et al. (2002) found puncture tests superior to chewing sounds and tensile measurements in forecasting sensory panelists' perception of texture traits. The Mohr ${ }^{\circledR}$ Digi-Test (Mohr and Associates, Richland, WA) computerized penetrometer captures data that correlate well with sensory firmness and sensory crispness by collecting constant velocity measurements (Evans et al., 2010). This is especially useful, because crispness has proven difficult to measure instrumentally with other devices. 
Establishing marker-locus-trait associations for texture traits depends on having an extensive, reliable phenotype database for traits of interest in breeding germplasm. Without high-quality phenotypic data, association statistics that link genomic sequences to traits cannot realize full potential (Bassil and Volk, 2010). Moreover, when standardized phenotyping protocols are used across several breeding programs, the resulting large data sets give more power to studies that detect and characterize quantitative trait loci (QTL) than would be had if each program conducted a smaller, isolated study.

A reference germplasm set of 467 individual genotypes including cultivars, selections, and seedlings was identified as part of the USDA-SCRI RosBREED project. The germplasm set provides efficient allelic representation of current parents in the large, publicly funded U.S. apple breeding programs of Cornell University (CU), Washington

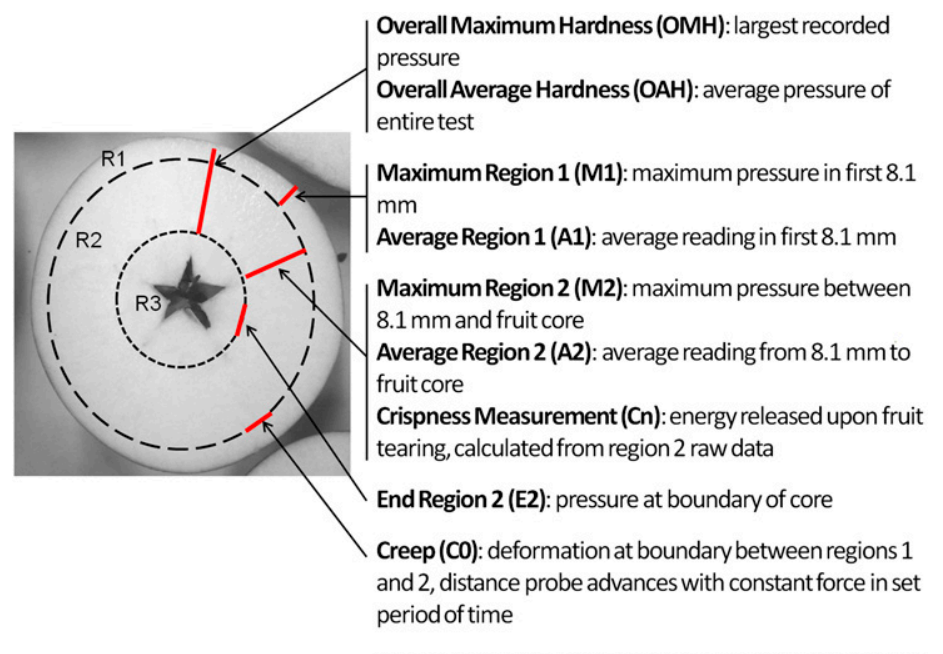

Quality Factor(QF): weighted index based on above traits

Fig. 1. Apple equatorial slice demonstrating MDT-1 fruit texture measures described by Evans et al. (2010) and Mohr and Mohr (2000). R1 is the outer area of the apple directly below the skin, R2 is the main edible portion of the fruit, and R3 contains the core. Red lines indicate regions in which traits are determined.
State University (WSU), and the University of Minnesota (UMN). Extensive phenotypic data, including instrumental and sensory measures of fruit texture, were collected on these individuals at each location in the years 2010 and 2011 under three regimes: at harvest, after 10 weeks of cold storage and 1 week at room temperature, and after 20 weeks of cold storage and 1 week at room temperature. Phenotypic data were collected adhering to a standardized protocol (Evans et al., 2012).

The objective in this article is to elaborate on methods used to obtain data on sensory and instrumental measures of fruit texture traits in the RosBREED apple Crop Reference Set (CRS) and describe variation and repeatability observed for these traits. We also report correlations between sensory and instrumental measures used in this study.

\section{Materials and Methods}

Plant material. The RosBREED apple CRS and supplementing individuals included 154 cultivars and parental selections as well as 313 seedlings of families chosen to provide efficient allelic representation of important breeding parents for a total of 467 related individuals. Subsets of the RosBREED CRS were grown at the UMN Horticultural Research Center near Chaska, MN, at the WSU Tree Fruit Research \& Extension Center in Wenatchee, WA, and at the CU New York State Agricultural Experiment Station in Geneva, NY. Evaluation of a reference

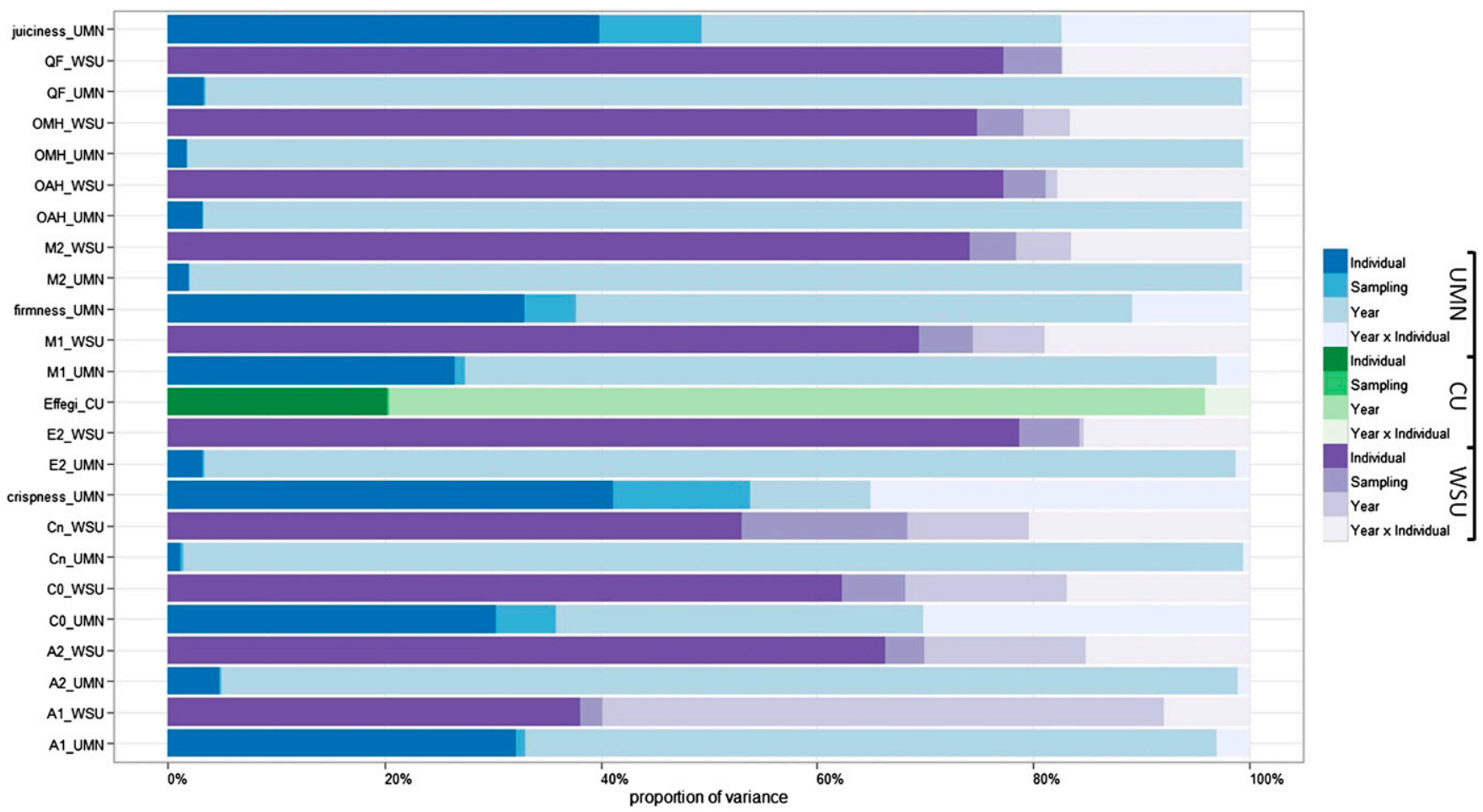

Fig. 2. Proportions of variance attributable to year, individual, sampling and year $\times$ individual for fruit texture measures at three locations at harvest. Analysis of variance was used. Data from University of Minnesota (UMN), Washington State University (WSU), and Cornell University (CU) are shown in shades of blue, purple, and green, respectively. Abbreviations are as follows: A1, A2 = average pressure regions 1 and 2 , respectively $(\mathrm{N})$; $\mathrm{C} 0=$ creep at boundary between regions 1 and $2(\mathrm{~cm}) ; \mathrm{Cn}=$ crispness measurement (derived value); 2 = pressure at core boundary $(\mathrm{N}) ; \mathrm{M} 1, \mathrm{M} 2=$ maximum pressure regions 1 and 2 , respectively $(\mathrm{N}) ; \mathrm{OAH}=$ overall average hardness $(\mathrm{N}) ; \mathrm{OMH}=$ overall maximum hardness $(\mathrm{N}) ; \mathrm{QF}=$ quality factor (derived value). The sensory measures of crispness, firmness, and juiciness were assessed on a 5-point scale. 
germplasm set would ideally include replication of all individuals on the same rootstock at each breeding location. In tree fruits, space and time limitations make such a design impractical. Most individuals, especially seedlings in breeding families, were not replicated within locations. As a result of winterhardiness and other factors, only one (2010) or eight (2011) cultivars were available for evaluation at more than one location. The trees were on several rootstocks and some seedlings were on their own roots. Trees were of various ages because they represented several generations of the breeding programs and different times of propagation. In addition to these potentially confounding factors, different personnel performed fruit quality evaluations at each location.

Phenotyping protocol. The complete RosBREED phenotyping protocol for apple is available at $<\mathrm{http}$ ://www.rosbreed.org/ sites/default/files/files/RosBREED_2010Phenotyping_protocol_Malus.pdf $>$, and in Evans et al. (2012). Portions of the protocol pertaining to fruit texture measurements are summarized below.

Cropload and fruit harvest. Because the amount of crop relative to tree size is known to affect some fruit quality traits (Stopar et al., 2002), cropload was managed so that young trees (third to sixth leaf) were thinned to 20 to 25 fruit per tree, whereas fruit on mature trees were thinned to four fruit per foot of branch length.

Fruit were monitored near the onset of the harvest season, and weekly inspection determined the most suitable harvest time for each individual so that all fruit were assessed at similar maturity. Maturity determination was based on a destructive starch-iodine reaction, described by Blanpied and Silsby (1992), and all fruit to be evaluated were harvested when the representative fruit tested in the orchard scored 3 or higher on their scale of 1 to 8 . As a result of variability across seasons and genotypes affecting starch accumulation and conversion, starch-iodine testing is an imperfect determinant of fruit maturity. When available, up to 20 sound fruit were harvested from one tree to obtain the 15 needed for evaluation. Extra fruit allowed for loss in storage. Fruits that were visibly damaged (e.g., sunburned, cracked, or rotting) or differing substantially from the average in size or maturity (e.g., fruit from the shaded tree center) were avoided. When 15 fruit were not available for harvest, all available, sound fruit were harvested.

Up to five fruit of each individual were evaluated at three points in time: harvest; 11 weeks postharvest in which the first 10 weeks were in cold storage and the last week was at room temperature (referred to as 10-week storage); and 21 weeks postharvest in which the first 20 weeks were in cold storage and the last week was at room temperature (referred to as 20-week storage). When fewer than 15 fruit were harvested, available fruit were evaluated at each time point until no more remained. For instance, if 10 fruit were available for an individual, the harvest and
10 -week evaluations were made and the 20week evaluation was omitted for lack of fruit.

Cold storage was at $1 \pm 2{ }^{\circ} \mathrm{C}$ in normal atmospheric conditions. Fruit were equilibrated to room temperature for $1 \mathrm{~d}$ before sensory and instrumental measures, when harvest evaluations could not be made the day of harvest. For storage evaluations, fruit were left at room temperature 1 week before evaluation.

Instrumental evaluation. All five fruit were marked to indicate sun-shade intermediate sides by drawing a line with a felttipped marker around the fruit where sun and shade skin coloring met. Before penetrometer assessment, a small disc of fruit peel was removed with a mandolin or knife at the apple equator on the marked line on either side of the apple. Only one side of each fruit was probed at the sun-shade interface, except at CU where both sides were probed with an Effegi penetrometer. Side selection was arbitrary at UMN and WSU, but bruised areas and other damage that may cause tissue softening were avoided. Fruit diameter, flesh firmness, and flesh crispness were measured (Evans et al., 2010) with a Mohr ${ }^{\circledR}$ Digi-Test (MDT-1; Mohr and Associates) penetrometer on factory settings at UMN and WSU. At CU, no instrumental crispness measure was available and fruit diameter was measured with a caliper.

The MDT-1 penetrometer collects data on several fruit texture parameters of interest. Traveling at a constant velocity, the penetrometer measures force required to push a plunger through the fruit flesh over two regions (Fig. 1). Region 1 is from point of entry to a depth of $\approx 8.1 \mathrm{~mm}$, representing the fruit cortex below the skin probed by many penetrometers (Mohr and Mohr, 2000). Region 2 , the main edible portion of the fruit, extends from the inside of boundary of Region 1 to the core. Force measures were recorded for the average, maximum and end point values of the two zones (Ax, Mx, and Ex, respectively). M1 (maximum force in region 1) is comparable to traditional industry penetrometer firmness determinants, and crispness $(\mathrm{Cn})$ is quantified as released energy as the probe advances (Mohr and Mohr, 2000). The quality factor (QF) is a weighted sum of several MDT1 traits, where high values suggest high fruit quality (Mohr and Mohr, 2000).
Sensory evaluation. Two trained sensory panelists at each location evaluated halved apple quarters of up to two fruit each for each individual tree when sufficient fruit were available. Slices for sensory evaluation were taken from the intermediate side, directly opposite the penetrometer site of insertion at UMN and WSU. At CU, sensory panelists were given the sun-side portion of the fruit. Firmness, crispness, and juiciness were rated on a 1 to 5 scale. For firmness, $1=$ very soft, $2=$ soft, $3=$ medium, $4=$ firm, and $5=$ very firm. Crispness was rated from $1=$ no noise to $5=$ very noisy. The juiciness scale was $1=$ dry, 2 = slightly juicy, $3=$ medium juicy, $4=$ juicy, and $5=$ very juicy. Sensory anchors for each trait were described by Evans et al. (2012); for instance, anchors for juiciness were the examples of banana and watermelon, scored 1 and 5, respectively. Panelists provided a single rating of each sensory parameter for each fruit sampled. Panelists held the peel of the apple slice and bit through the fruit cortex to determine crispness and chewed for firmness and juiciness assays. A second slice of the same apple quarter was available for confirmation. When fewer than four fruit were available, fruit were divided between panelists. Using the 5-point scale for each sensory trait, a single score for each individual in the germplasm set was reported in each year it was evaluated at a location. At UMN, the mean scores of the four fruit, two by each panelist, were reported, and at $\mathrm{CU}$ and WSU, panelists discussed their ratings and reported a consensus score. CU and WSU panelists evaluated one or more fruit each until a consensus score was verbally agreed on. Changes in sensory traits were calculated for each individual as the difference between ratings for a trait at harvest evaluation and after storage (e.g., 10-week storage rating minus the harvest rating).

Statistical analyses. Harvest texture traits from individuals having more than one fruit in both seasons were analyzed by analysis of variance (ANOVA) with years and individuals as crossed factors. Years were treated as random effects and individuals were treated as fixed effects. Values for each individual fruit were used in the ANOVA to determine fruit sampling contribution to variance in the diverse texture measurements. Up to five fruit were sampled for each instrumental trait, and

Table 1. Counts of individuals phenotyped by year, location, and storage duration for sensory and instrumental traits at the University of Minnesota (UMN), Washington State University (WSU) and Cornell University (CU).

\begin{tabular}{|c|c|c|c|c|c|c|}
\hline & \multicolumn{3}{|c|}{2010} & \multicolumn{3}{|c|}{2011} \\
\hline & Harvest & $\begin{array}{l}\text { 10-week } \\
\text { storage }\end{array}$ & $\begin{array}{l}20 \text {-week } \\
\text { storage }\end{array}$ & Harvest & $\begin{array}{l}\text { 10-week } \\
\text { storage }\end{array}$ & $\begin{array}{l}\begin{array}{l}20 \text {-week } \\
\text { storage }\end{array} \\
\text { a }\end{array}$ \\
\hline \multicolumn{7}{|l|}{ Sensory } \\
\hline UMN & 73 & 34 & 23 & 176 & 144 & 135 \\
\hline WSU & 90 & 83 & 79 & 98 & 93 & 87 \\
\hline $\mathrm{CU}$ & 51 & 37 & 35 & 56 & 54 & 50 \\
\hline Total & 214 & 154 & 137 & 330 & 291 & 272 \\
\hline \multicolumn{7}{|c|}{ Instrumental } \\
\hline UMN & 76 & 35 & 25 & 171 & 146 & 137 \\
\hline WSU & 91 & 83 & 79 & 98 & 91 & 87 \\
\hline $\mathrm{CU}$ & 49 & 36 & 33 & 56 & 55 & 50 \\
\hline Total & 216 & 154 & 137 & 325 & 292 & 274 \\
\hline
\end{tabular}




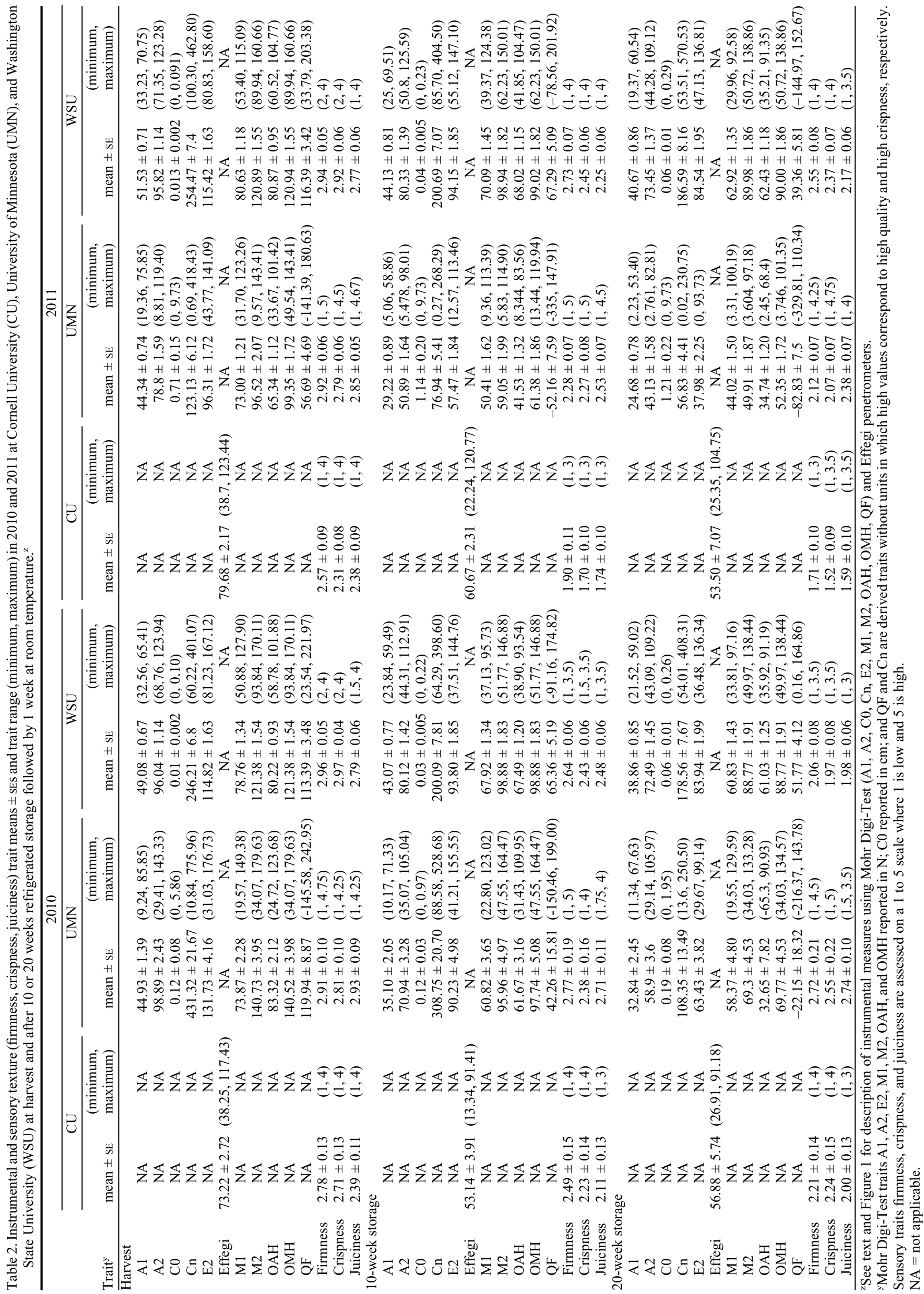


four fruit were sampled for each sensory trait. Data for 46 individuals evaluated in both years were available from UMN, of which 17 individuals were seedlings, 22 were selections and seven were cultivars ['Akane', 'Arlet', 'Gingergold', 'Honeycrisp', 'Sawa', Snowsweet ${ }^{\circledR}$ (cultivar Wildung), and Zestar! ${ }^{\circledR}$ (cultivar Minnewashta)]. Data for 105 individuals, comprising 69 seedlings, 17 selections, and 19 cultivars ['Ambrosia', '8S6923' (Aurora Golden Gala' ${ }^{\mathrm{TM}}$ ), 'Cameo', 'Co-op 39' (Crimson Crisp $\left.{ }^{\top M}\right)$, 'Cripps Pink' (Pink Lady ${ }^{\circledR}$ ), 'Delblush', 'Delorgue', 'Enterprise', 'Goldrush', 'Hatsuaki', 'SPA440' (Nicola $\left.{ }^{\mathrm{TM}}\right)$, 'Pinova' (Piñata $^{\circledR}$, Corail $\left.^{\mathrm{TM}}\right)$, 'Scifresh' $\left(\mathrm{Jazz}^{\mathrm{TM}}\right)$, 'Scired'(Pacific Queen ${ }^{\mathrm{TM}}$ ), 'Sciros'(Pacific Rose $^{\mathrm{TM}}$ ), 'Silken', 'Nevson' (Sonya ${ }^{\mathrm{TM}}$ ), 'Coop 29' (Sundance ${ }^{\mathrm{TM}}$ ), and 'Cripps Red' (Sundowner $\left.{ }^{\circledR}\right)$ ] were available from both years at WSU. In both years at $\mathrm{CU}$, data for 36 individuals - 32 seedlings, one selection, and three cultivars ('Hudson', 'Russian Seedling', and 'SunCrisp') — were available. The proportions of total variance accounted for by year, individual, year $x$ individual interaction, and variation among fruit from an individual were determined for instrumental measurements at all locations and for sensory evaluations at UMN.

For all other statistical analyses, means of the five fruit were used to represent each individual at a location, year, and storage duration. Ranges, means, and SEs were determined for instrumental and sensory texture traits for each location-year-storage duration instance. Because individuals, sensory panelists, and environmental factors within location effects were confounded, means separation would not be informative. Spearman's rank order correlations were determined between non-parametric sensory measures and between instrumental and sensory measures at each location-year-storage instance. Year-toyear repeatability of sensory and instrumental texture measures at each location-storage treatment were estimated using Pearson's product-moment correlation. All statistical analyses were conducted using R (R Development Core Team, 2011) and bean plot graphs were constructed using the beanplot package (Kampstra, 2008) in R. Bean plots for each year included data from individuals assessed in both years and individuals assessed in only 2010 or 2011.

\section{Results and Discussion}

In 2010 and 2011, fruit from 216 and 330 individuals, respectively, were harvested and a total of 369 individuals of the CRS were evaluated over the two years. During the 2010 harvest, 73, 90, and 51 individuals were evaluated at UMN, WSU, and $\mathrm{CU}$, respectively, for sensory texture. In 2011, 176, 98, and 56 individuals were evaluated at UMN, WSU, and CU, respectively. Postharvest attrition of an individual's fruit during storage varied with location and year (Table 1). Attrition reflected fruit availability and/or storage potential of the individual in some instances. Frost injury at bloom in 2010 reduced the number of fruit available at $\mathrm{CU}$. A 2010 summer hailstorm likely caused the high attrition from harvest to first storage evaluation at UMN, where fruit samples were limited in availability.

Means, ranges, and phenotypic variances of all individuals at a location differed among locations for some traits (Table 2), reflecting the largely unique set of individuals and differing environmental conditions at each location. Average sensory scores diverged little among locations. The majority of texture trait scores and measures decreased in magnitude with increasing storage duration. Higher sensory scores were observed at UMN and WSU at harvest and after the 10-week storage duration than for the 20 -week storage treatment. MDT-1 instrumental measures were more acutely different between WSU and UMN means in 2011 than in 2010. This result could be the result of marked differences in environmental conditions those years or a reflection of the specific individual genotypes available in each harvest year. Observed instrumental trait ranges were broader for many MDT-1 measures at UMN compared with WSU in 2010

The individuals considered exhibited a wide range of fruit firmness. Average instrumental firmness (MDT-1 M1 and Effegi penetrometer) of a five-fruit sample representing an individual ranged from 19.6 to $149.4 \mathrm{~N}$ at harvest, 9.4 to $124.4 \mathrm{~N}$ after 10 weeks of cold storage, and 3.3 to $129.6 \mathrm{~N}$ after 20 weeks of cold storage. Averaging harvest and two-month storage treatment measures, Evans et al. (2010) reported M1 values ranging from $\approx 40$ to $100 \mathrm{~N}$ for cultivars including 'Sciearly' (Pacific Beauty ${ }^{\top \mathrm{M}}$ ), 'Braeburn', 'Cripps Pink', 'Fuji', 'Honeycrisp', and others. Using Magness-Taylor, Effegi, and other penetrometers, under treatments varying from three to 14 months of storage, DeLong et al. (2000) reported firmness ranges from 35 to $75 \mathrm{~N}$ for the cultivars Cortland, McIntosh, and Northern Spy. Higher and especially lower values than those reported for commercial cultivars were expected in the apple CRS that was twothirds constituted of unselected breeding germplasm.

ANOVA indicated that the proportion of variance attributable to fruit sampling, individual, year, and year $\times$ individual depended on location and trait of interest (Fig. 2). The effect of year was a much lesser contributor to texture variation at WSU than at $\mathrm{CU}$ and UMN.

Repeatability of sensory texture traits was generally low to moderate with Pearson's correlation coefficients comparing 2010 and 2011 data ranging from 0.13 to 0.78 for sensory crispness (WSU at harvest and UMN at 20 -week storage, respectively) and 0.30 to 0.81 for sensory firmness (CU at harvest and UMN at 20-week storage, respectively; Table $3)$. Pearson's correlation coefficients ranged from 0.19 to 0.70 for sensory juiciness (CU at

Table 3. Year-to-year repeatability (shown by Pearson's correlations) of sensory (firmness, crispness, juiciness) and instrumental texture traits measured at harvest and after 10 or 20 weeks refrigerated storage followed by 1 week at room temperature at the University of Minnesota (UMN), Washington State University (WSU), and Cornell University (CU). ${ }^{\mathrm{z}}$

\begin{tabular}{|c|c|c|c|c|}
\hline Trait $^{y}$ & Location & Harvest & 10-week storage & 20-week storage \\
\hline \multirow[t]{2}{*}{$\overline{\mathrm{A} 1}$} & UMN & $0.64 * * * *$ & $0.67 * * *$ & $0.88 * * * *$ \\
\hline & WSU & $0.65 * * * *$ & $0.72 * * * *$ & $0.71 * * * *$ \\
\hline \multirow[t]{2}{*}{$\mathrm{A} 2$} & UMN & $0.54 * * * *$ & $0.63 * * *$ & $0.86 * * * *$ \\
\hline & WSU & $0.62 * * * *$ & $0.66 * * * *$ & $0.72 * * * *$ \\
\hline \multirow[t]{2}{*}{$\mathrm{C} 0$} & UMN & $0.39 * *$ & $-0.16 \mathrm{NS}$ & $-0.11 \mathrm{NS}$ \\
\hline & WSU & $0.42 * * *$ & $0.73 * * * *$ & $0.68 * * * *$ \\
\hline \multirow[t]{2}{*}{$\mathrm{Cn}$} & UMN & $0.47 * * *$ & $0.33 \mathrm{NS}$ & $0.29 \mathrm{NS}$ \\
\hline & WSU & $0.37 * * *$ & $0.61 * * * *$ & $0.49 * * * *$ \\
\hline \multirow[t]{2}{*}{ E2 } & UMN & $0.49 * * * *$ & $0.54 * *$ & $0.85 * * * *$ \\
\hline & WSU & $0.68 * * * *$ & $0.66 * * * *$ & $0.73 * * * *$ \\
\hline \multirow[t]{2}{*}{ M1 } & UMN & $0.62 * * * *$ & $0.71 * * * *$ & $0.93 * * * *$ \\
\hline & WSU & $0.54 * * * *$ & $0.65 * * * *$ & $0.67 * * * *$ \\
\hline \multirow[t]{2}{*}{ M2 } & UMN & $0.49 * * * *$ & $0.53 * *$ & $0.84 * * * *$ \\
\hline & WSU & $0.66 * * * *$ & $0.66 * * * *$ & $0.72 * * * *$ \\
\hline \multirow[t]{2}{*}{ QF } & UMN & $0.54 * * * *$ & $0.64 * * *$ & $0.85 * * * *$ \\
\hline & WSU & $0.61 * * * *$ & $0.74 * * * *$ & $0.48 * * *$ \\
\hline Effegi & $\mathrm{CU}$ & $0.70 * * * *$ & $0.71 * * * *$ & $0.73 * * *$ \\
\hline \multirow[t]{3}{*}{ Crispness } & UMN & $0.48 * * *$ & $0.56 * *$ & $0.78 * * * *$ \\
\hline & WSU & $0.13 \mathrm{NS}$ & $0.37 * *$ & $0.66 * * * *$ \\
\hline & $\mathrm{CU}$ & 0.14 NS & $0.65 * * *$ & $0.59 * *$ \\
\hline \multirow[t]{3}{*}{ Firmness } & UMN & $0.43 * * *$ & $0.75 * * * *$ & $0.81 * * * *$ \\
\hline & WSU & $0.37 * * *$ & $0.37 * *$ & $0.46 * * *$ \\
\hline & $\mathrm{CU}$ & $0.30 \mathrm{NS}$ & $0.54 * *$ & $0.44^{*}$ \\
\hline \multirow[t]{3}{*}{ Juiciness } & UMN & $0.40 * *$ & $0.40 *$ & $0.29 \mathrm{NS}$ \\
\hline & WSU & $0.29 * *$ & $0.38 * *$ & $0.35 * *$ \\
\hline & $\mathrm{CU}$ & $0.19 \mathrm{NS}$ & $0.65 * * *$ & $0.70 * * *$ \\
\hline
\end{tabular}

${ }^{\mathrm{z}}$ Data aggregated for all genotypes at each location. See text and Figure 1 for description of instrumental measures using Mohr Digi-Test (A1, A2, C0, Cn, E2, M1, M2, OAH, OMH, QF) and Effegi penetrometers. ${ }^{y}$ Mohr Digi-Test traits A1, A2, E2, M1, M2, OAH, and OMH reported in N; C0 reported in cm; and QF and $\mathrm{Cn}$ are derived traits without units, whereas high values correspond to high quality and high crispness, respectively. Sensory traits firmness, crispness, and juiciness are assessed on a 1 to 5 scale, with 1 corresponding to low and 5 to high.

NS, $* * *, * * *, * * * *$ Nonsignificant and significant at $P \leq 0.05,0.01,0.001$, or 0.0001 , respectively. 


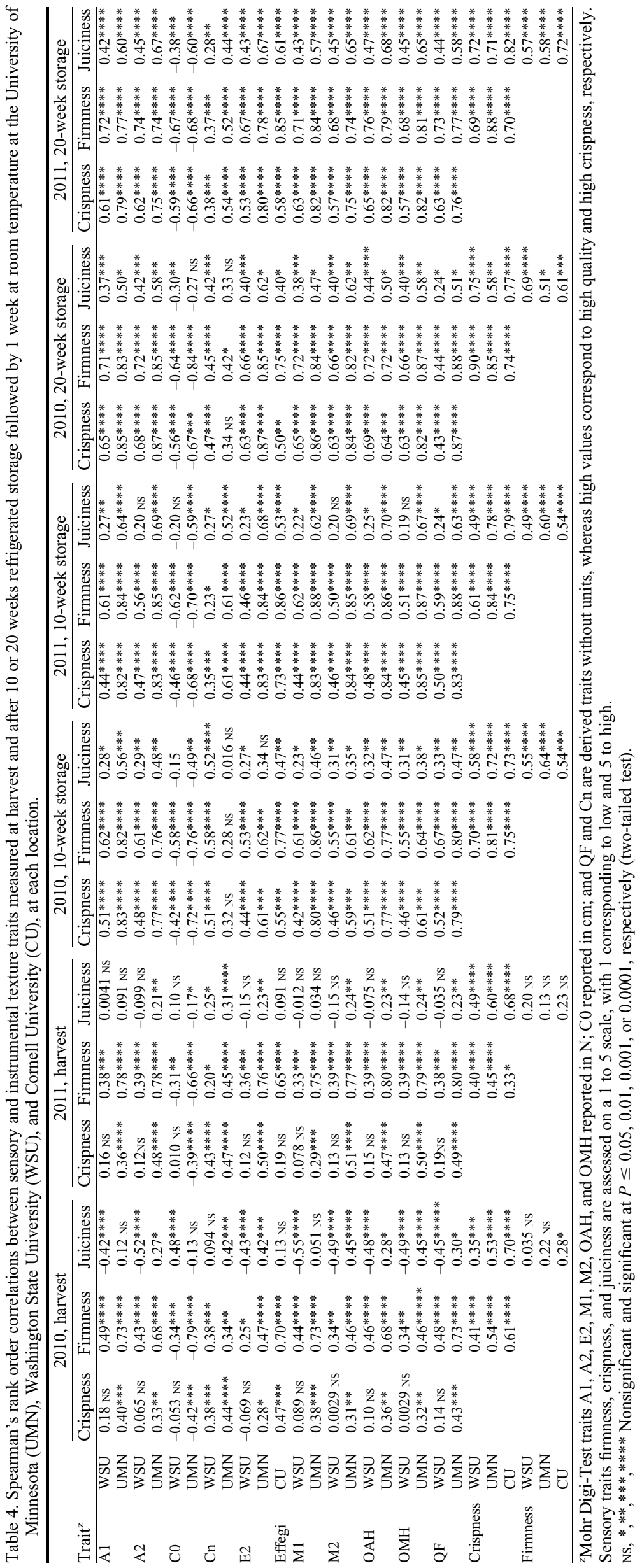

harvest and CU at 20-week storage, respectively). Year-to-year repeatability tended to be higher for instrumental measures than sensory measures (Table 3). Year-to-year correlations at WSU and UMN, as measured by the MDT-1 penetrometer, were moderate with statistically significant correlations ranging from 0.37 to 0.93 . Between-year correlations in instrumental firmness, as measured with the Effegi penetrometer at CU, were 0.70, 0.71 , and 0.73 , chronologically from harvest through storage.

High trait repeatability across years indicates low genotype $\times$ year interaction relative to variation among individuals for a trait. Therefore, response to selection on highly repeatable traits with MAS is expected to be larger than phenotypic selection without markers, even when marker selection is based on associations observed from only a few years of data. Although markers for quantitative traits that are relatively stable across years in their expression will be easier to develop and validate, markers for traits affected more by yearly variation may be of higher use than stable markers, because selection based on phenotype alone will be less reliable.

Correlations across years between sensory and instrumental traits indicated consistent effects of factors affecting these relationships (Table 4). In some instances, correlations between sensory and instrumental texture measures were high, especially after 20 weeks of storage. For instance, the MDT-1 A2 measure showed correlations to sensory measures ranging from 0.42 to 0.87 after 20 weeks of storage. The MDT-1 M1 correlated well with sensory firmness. Spearman's rank correlations for MDT-1 M1 and sensory firmness measurements at harvest at UMN were 0.73 and 0.75 in 2010 and 2011, respectively. Evans et al. (2010) observed a correlation of 0.66 between M1 and sensory hardness in fruit from central Washington. With respect to sensory traits, crispness was moderately correlated with firmness (ranging from 0.33 to 0.61 at harvest) and with juiciness ( 0.35 to 0.70 at harvest), but juiciness was poorly correlated with firmness ( 0.04 to 0.28 at harvest). Differences in individuals and sensory panelists likely account for some observed differences in strength of correlations among locations, such as the contrast between harvest MDT-1 M1 to crispness correlations between WSU and UMN (Table 4).

The MDT-1 measures A1, M1, and QF were especially predictive of sensory firmness (Table 4). MDT-1 C0 was strongly and negatively correlated with firmness (Table 4). The use of the MDT-1 to predict sensory measures appears to be location-dependent at harvest, because WSU measurements did not show nearly as strong correlations as UMN data. The average sensory to instrumental correlation at UMN was 0.49 , whereas the average at WSU was 0.29 . The MDT-1 Cn, proposed to measure crispness, was similarly correlated with sensory firmness and sensory crispness in this study (average correlations of 0.40 and 0.43 , respectively, across locations 
and years). Therefore, the choice to replace sensory evaluation in breeding with a MDT-1 penetrometer may not be recommended for all locations or selection purposes.

Changes in sensory and instrumental trait values from harvest to 10 weeks storage and from harvest to 20 weeks storage were compared visually (Figs. 3 and 4). Statistical comparisons would be complex and not be very informative because few individuals were common across locations as well as years. Additionally, not all individuals evaluated at harvest had sufficient fruit to be analyzed after storage. As expected, larger changes in texture occurred after 20 weeks of storage than after 10 weeks of storage. An exception was 2010 instrumental firmness at CU where the average firmness loss from harvest to 10 weeks storage was $24 \mathrm{~N}$ but loss from harvest to 20 weeks storage was $21 \mathrm{~N}$ (bold, black bars mark distribution means, Fig. 4). Rapidly softening fruit representing some individuals may not have been available at the last evaluation, producing this result. Completely decayed fruit were discarded because they were unsuitable for sensory panelist consumption. Differences in distributions among locations demonstrate that although a standardized protocol with a common scale and anchors was agreed on, sensory scores represent a more subjective and less repeatable evaluation system than instrumental evaluation. Standardized protocols greatly increase the usefulness of sensory panel evaluation. On average, sensory scores dropped less than 1 point on the 1 to 5 scale after storage from harvest to 20 weeks, whereas instrumental measures detected average firmness losses of $24 \%$ to $34 \%$ after 20 weeks of storage. The relative changes after 20 weeks of storage in sensory and instrumental crispness scores were similar (Table 2, distributions not presented) with sensory scores declining $18 \%$ to $28 \%$ and Cn declining $21 \%$ to $46 \%$ at WSU and UMN. In the majority of instances, sensory evaluation detected a proportionally smaller texture loss than that detected by instrumental evaluation.

When using these data for QTL discovery, it is important to consider that harvest sensory scores are not directly comparable to sensory scores later in the experiment. The percent loss in texture measured instrumentally was in most instances greater than that perceived by panelists. Differences in texture distributions between years within a location were primarily differences of distribution shape. Distribution shape differences between years at the same location could be the result of different sets of individuals evaluated between the years, environmental effects, or a change in scoring regimen (e.g., WSU panelists agreed on a single, integer score in 2011 rather than verbally averaging scores like in 2010).

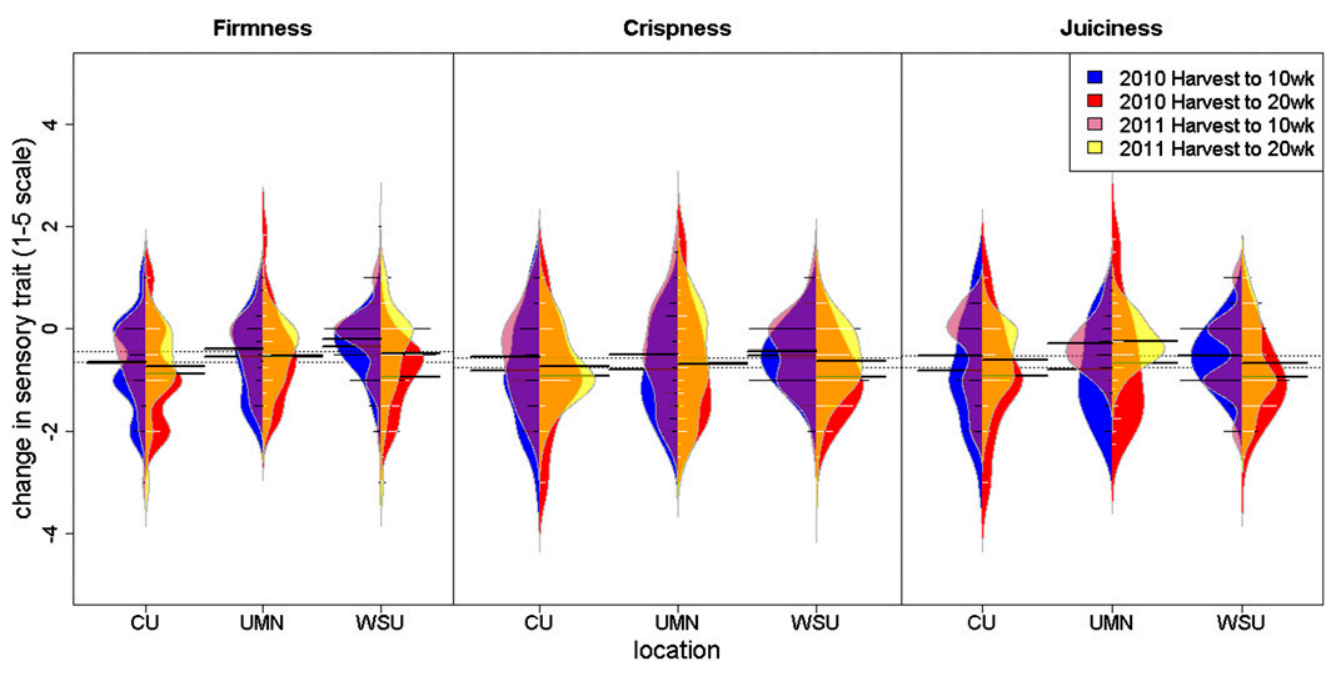

Fig. 3. Bean plots of changes in sensory texture components from harvest to 10 weeks of storage (distributions to left of vertical lines) and from harvest to 20 weeks storage (distributions to the right of vertical lines) at University of Minnesota (UMN), Washington State University (WSU), and Cornell University (CU). Dashed horizontal lines mark year means across locations. Bold black horizontal lines mark the mean of each storage interval distribution. Minor black and white horizontal lines are individual observations, in which line width indicates multiple observations of the same value, in the format of a histogram, although filled areas are a density trace of the distribution. Sensory measures were assessed on a 5-point scale.

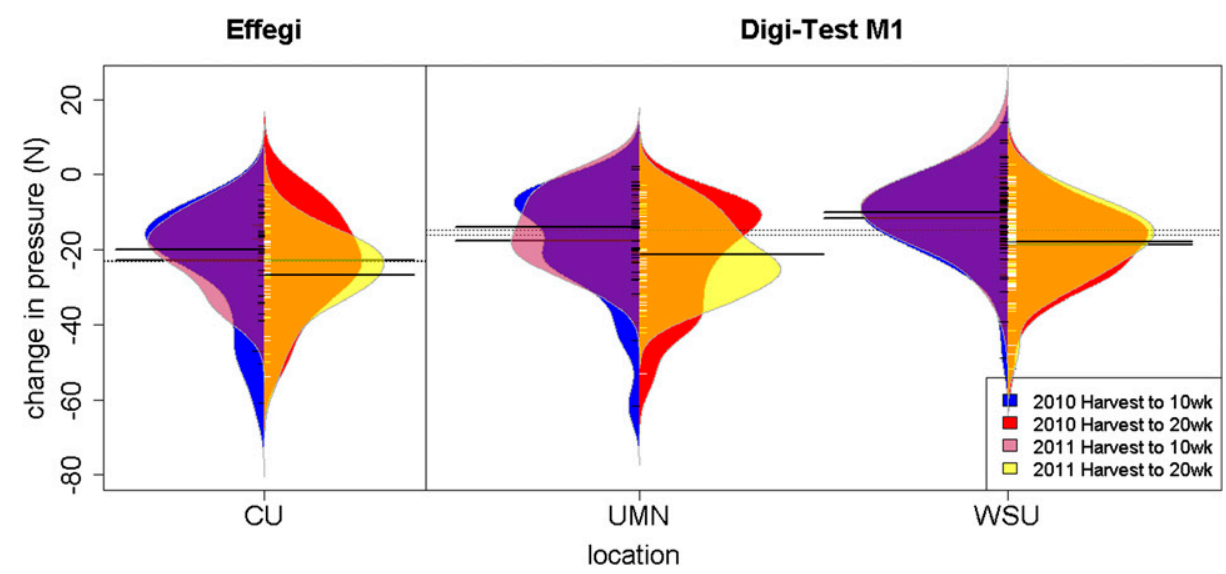

Fig. 4. Observed changes in instrumental firmness over storage at University of Minnesota (UMN), Washington State University (WSU), and Cornell University (CU). Dashed horizontal lines mark year averages across locations. Bold black horizontal lines mark the mean of each distribution. Minor black and white horizontal lines are individual observations, in which line width indicates multiple observations of the same value, in the format of a histogram, whereas filled areas are a density trace of the distribution. M1 measured by Mohr Digi-Test-1 (Fig. 1) is reportedly equivalent to firmness measured with an Effegi penetrometer (Mohr and Mohr, 2000). 


\section{Conclusions}

This article presents information on fruit texture trait distributions in a reference germplasm set of three breeding programs that can be used to simultaneously detect and validate QTL. The wide ranges of observed phenotypic values for these traits is a prerequisite for detecting marker-trait-locus associations. As a result of the lack of replication of many individuals across sites, these data should not be directly pooled for QTL detection, because confounded sources of variation at a location (orchard environment, instruments, and sensory panelists) have not yet been accounted for in this study. QTL detected at multiple locations, through independent analyses of each location, would be of particular interest as a result of robustness across the confounded factors. The data may be adjusted for confounding factors using relatedness among individuals among locations, resulting in a larger population for examining location effects and for pedigree-based QTL analysis. Knowledge of QTL $\times$ environment interactions, especially rank-order shifts in which a QTL effect is ranked of higher use in one environment than in others, will be pertinent to deployment of MAB and MAS that targets apple production environments beyond those of the three research facilities.

Marked differences among locations in trait ranges for instrumental texture measures may affect the detection of QTL or their effect magnitudes at each location. Germplasm fixed or nearly fixed for extreme-effect alleles at a QTL will have less phenotypic variation for the trait, perhaps leading to the discovery of lesser-effect QTL but also limiting the ability to detect the large-effect QTL. When predictive markers are developed for application outside the initial germplasm studied, ascertainment bias may limit marker use, because not all alleles of use may be present in the initial study. The analysis of phenotypic variation in these three diverse breeding programs across years can help with interpretation of QTL analyses that will follow. Moreover, an understanding of trait variability across years is essential in recommending markers for MAB.

Other researchers may use RosBREED's standardized phenotyping protocols on additional germplasm or years to supplement the original data set to develop a more robust interpretation or to examine the effects of changing one or more parameters. Individuals in this set have been genotyped with the
International RosBREED SNP Consortium (IRSC) 8K SNP array developed by Chagné et al. (2012). Phenotypic and genotypic data for this reference germplasm will be curated and available for use by the international community of apple breeders and allied scientists for QTL mapping and validation as well as other analyses through the Breeders Toolbox application at the Genome Database for Rosaceae $(<\mathrm{http}: / /$ www.rosaceae.org $>$ ).

Because the phenotyping protocol used here is similar to that of the European Highquality Disease Resistant Apples for Sustainable Agriculture project (Evans et al., 2012), a meta-analysis could be very informative. Similar fruit texture parameters may also be evaluated in closely related crops to better understand the traits, to find widely predictive markers, or to generate information about synteny among the respective genomes.

\section{Literature Cited}

Bassil, N.V. and G.M. Volk. 2010. Standardized phenotyping: Advantages to horticultureIntroduction to the workshop. HortScience 45:1306.

Blanpied, G.D. and K.J. Silsby. 1992. Predicting harvest date windows for apples. Cornell Coop. Ext. Info. Bul. 221:1-12.

Bliss, F.A. 2010. Marker-assisted breeding in horticultural crops. Acta Hort. 859:339-350.

Brookfield, P.L., S. Nicoll, F.A. Gunson, F.R. Harker, and M. Wohlers. 2011. Sensory evaluation by small postharvest teams and the relationship with instrumental measures of apple texture. Postharvest Biol. Technol. 59:179186.

Chagné, D., R.N. Crowhurst, M. Troggio, M.W. Davey, B. Gilmore, C. Lawley, S. Vanderzande, R.P. Hellens, S. Kumar, A. Cestaro, R. Velasco, D. Main, J.D. Rees, A. Iezzoni, T. Mockler, L. Wilhelm, E. van de Weg, S.E. Gardiner, N. Bassil, and C. Peace. 2012. Genome-wide SNP detection, validation, and development of an $8 \mathrm{k}$ SNP array for apple. PLoS ONE 7:e31745.

DeLong, J.M., R.K. Prange, P.A. Harrison, and K.B. McRae. 2000. Comparison of a new apple firmness penetrometer with three standard instruments. Postharvest Biol. Technol. 19:201209.

Evans, K., L. Brutcher, B. Konishi, and B. Barritt. 2010. Correlation of sensory analysis with physical textural data from a computerized penetrometer in the Washington State University apple breeding program. HortTechnology 20:1026-1029.

Evans, K., J. Luby, S. Brown, M. Clark, Y. Guan, B. Orcheski, C. Schmitz, C. Peace, E. van de Weg, and A. Iezzoni. 2012. Large-scale standardized phenotyping of apple in RosBREED.
Proc. 4th Intl. Conf. Postharv. Unlimited 2011. Acta Hort. 945:233-238.

Fillion, L. and D. Kilcast. 2002. Consumer perception of crispness and crunchiness in fruits and vegetables. Food Qual. Prefer. 13:23-29.

Harker, F.R., R.L. Amos, G. Echeverría, and F.A. Gunson. 2006. Influence of texture on taste: Insights gained during studies of hardness, juiciness, and sweetness of apple fruit. J. Food Sci. 71:S77-S82.

Harker, F.R., F.A. Gunson, and S.R. Jaeger. 2003. The case for fruit quality: An interpretive review of consumer attitudes, and preferences for apples. Postharvest Biol. Technol. 28:333-347.

Harker, F.R., E.M. Kupferman, A.B. Marin, F.A. Gunson, and C.M. Triggs. 2008. Eating quality standards for apples based on consumer preferences. Postharvest Biol. Technol. 50: $70-78$.

Harker, F.R., J. Maindonald, S.H. Murray, F.A. Gunson, I.C. Hallett, and S.B. Walker. 2002. Sensory interpretation of instrumental measurements 1: Texture of apple fruit. Postharvest Biol. Technol. 24:225-239.

Ioannides, Y., M.S. Howarth, C. Raithatha, M. Defernez, E.K. Kemsley, and A.C. Smith. 2007. Texture analysis of Red Delicious fruit: Towards multiple measurements on individual fruit. Food Qual. Prefer. 18:825-833.

Kampstra, P. 2008. Beanplot: A boxplot for alternative for visual comparison of distributions. J. Stat. Software 28:1-9. 23 Nov. 2012. <http:// www.jstatsoft.org/v28/c01/>.

McKay, S.J., J.M. Bradeen, and J.J. Luby. 2011. Prediction of genotypic values for apple fruit texture traits in a breeding population derived from 'Honeycrisp'. J. Amer. Soc. Hort. Sci. 136:408-414.

Mohr, B.C. and C.L. Mohr. 2000. The Mohr DigiTest (MDT) computerized agricultural penetrometer as an apple maturity tool. $30 \mathrm{Apr} .2012$. $<\mathrm{http}: / /$ www.mohr-engineering.com/documents/ MDTPosterPaper.pdf $>$.

R Development Core Team. 2011. R: A language and environment for statistical computing. $\mathrm{R}$ Foundation for Statistical Computing, Vienna, Austria. 23 Nov. 2012. <http://www.R-project. org/>.

Roudaut, G., C. Dacremont, B. Vallés Pàmies, B Colas, and M. Le Meste. 2002. Crispness: A critical review on sensory and material science approaches. Trends Food Sci. Technol. 13:217-227.

Stopar, M., U. Bolcina, A. Vanzo, and U. Vrhovsek. 2002. Lower crop load for cv. Jonagold apples (Malus $\times$ domestica Borkh.) increases polyphenol content and fruit quality. J. Agr. Food Chem. 50:1643-1646.

Vickers, Z. and M.C. Bourne. 1976. A psychoacoustical theory of crispness. J. Food Sci. 41:1158-1164.

Zdunek, A., J. Cybulska, D. Konopacka, and K. Rutkowski. 2011. Inter-laboratory analysis of firmness and sensory texture of stored apples. Intl. Agrophysics 25:67-75. 